\title{
Fault Diagnosis and Isolation for Brushless Electronic Returnless Fuel Systems
}

\author{
Yao $\mathrm{Hu}^{1}$ \\ ${ }^{1}$ General Motors, Warren, Michigan, 48092, USA \\ yao.hu@gm.com
}

\begin{abstract}
The brushless electronic returnless fuel system (ERFS) is a complex system, which is subject to different faults such as motor resistance increase and pressure sensor bias. Any of these faults may lead to no-start and safety-related issues such as loss of engine power. It's challenging to develop a diagnosis and isolation method to detect and isolate faults in the field, with feature signals which capture different fault signatures. In this study, we first develop a set of feature signals which are used to monitor the behavior, track the degradation, predict the potential failures, and diagnose the issues of the brushless ERFS. The feature signals include the estimated resistance, current error ratio, PWM duty cycle error ratio, zero pump speed ratio, etc. Then we develop a fault diagnosis and isolation algorithm to generate the diagnostic results based on feature signals. The algorithm is tested on a vehicle with fault injection. The results show that different faults can be identified correctly with the developed algorithm. This study enables development of diagnosis and prognosis for the brushless ERFS, which can protect customers from walk-home scenarios and safety related issues due to the brushless ERFS failures.
\end{abstract}

\section{INTRODUCTION}

In an internal combustion engine (ICE) vehicle, the brushless electronic returnless fuel system (ERFS) pushes the fuel from the fuel tank to the fuel feed pipe, feeding the fuel to the highpressure fuel pump, which generates a high pressure fuel flow going to the fuel rails and injectors. Figure 1 shows the structure of the brushless ERFS. To control the brushless fuel pump, the engine control module (ECM) first determines the commanded pump speed based on the desired fuel pressure under current vehicle operation. Then the fuel tank zone module (FTZM) determines the 3-phase PWM based on the commanded pump speed, which controls the inverter to drive the brushless fuel pump.

\footnotetext{
Yao Hu. This is an open-access article distributed under the terms of the Creative Commons Attribution 3.0 United States License, which permits unrestricted use, distribution, and reproduction in any medium, provided the original author and source are credited.
}

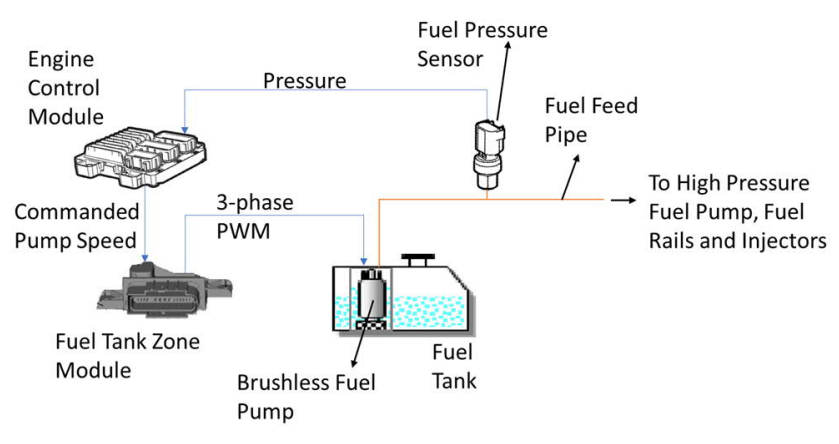

Figure 1. Brushless ERFS.

The brushless ERFS is critical since its failures can lead to no-start and some safety-related issues such as loss of engine power. The brushless ERFS failure modes include motor failures, fuel pressure sensor failures, pump leakage, controller failures, etc. To identify potential failures in advance, and alert customers to avoid the severe scenarios such as walk-home, it is important to develop the diagnosis and prognosis (DnP) system of the brushless ERFS. In the DnP system, the fault diagnosis and isolation algorithms identify the fault type by analyzing data which represents the operations of the brushless ERFS.

Many studies on fault diagnosis and isolation are on the application in bearing and rotating machinery. Tax, Ypma, and Duin (1999) apply a support vector data description method on vibration signals to diagnose surface cracking of gears in pumps. Lu, Wang, Ragulskis, and Cheng (2016) propose an image processing method to diagnose faults in rotating machinery. Vibration signal is transformed into a bispectrum contour map, and the speeded-up robust feature (SURF) is used to extract fault features. Wen, $\mathrm{Li}, \mathrm{Gao}$, and Zhang (2018) convert the time domain signals into 2D images and applies a convolutional neural network to perform diagnosis. The method is tested on bearing and pump systems. Majority of these studies requires high sampling rate of signals, and frequency domain or time-frequency domain analysis.

There are also some studies focused on diagnosis of brushless DC motor. Moseler and Isermann (2000) present a method to 
estimate parameters such as resistance and back EMF coefficient based on an analytical model. Based on the estimated parameters, faults of resistance increase and friction increase are detected. Sun, Chai, Su, Zhu, and Luo (2014) use a neural network to model the motor system. With adaptive lifting wavelet decomposition, the error between the actual speed and the estimated speed from the neural network is analyzed to detect faults such as voltage leak, phase fault, load error and shake error. Many studies discuss the diagnosis of switch fault in the inverter. Most of them utilize analysis in frequency domain. Tashakori and Ektesabi (2013) present a method using spectral energy density based on discrete Fourier transform (DFT) on voltage signal to diagnose switch faults. Diagnosis of stator-winding inter-turn short circuits is discussed by Awadallah, Morcos, Gopalakrishnan, and Nehl (2005). DFT and short-time Fourier transform are performed on voltage and torque signals to identify the number and location of short turns, respectively. These studies are focused on specific faults and develop features based on them.

The fault diagnosis and isolation for brushed ERFS is discussed in several patents. Ghoneim (2013) discusses a method to estimate the motor speed at steady state to monitor the state of health of the brushed ERFS. Ghoneim (2014) introduces a method of fault isolation based on estimation of resistance, back EMF coefficient, current ratio, etc. Ghoneim and Howell (2016) use lookup tables to estimate current and motor speed based on voltage and fuel pressure to detect faults. These methods use the analytical model of brushed DC motor to monitor the behavior of the brushed ERFS and diagnose its faults.

Due to the challenges of low sampling rate of signals and unique failure modes in the brushless ERFS, we propose a new solution based on the analytical model of the brushless DC motor. This work is focused on the faults of brushless fuel pump and the fuel pressure sensor on the fuel feed pipe. To capture the fault signatures, we determine a set of feature signals including estimated resistance, current error ratio, PWM duty cycle error ratio, zero pump speed ratio, etc. Moreover, a diagnostic algorithm is developed to check the behaviors of these signals to determine the fault type.

In Section 2, the details of the feature generation algorithm are discussed. Section 3 introduces the diagnostic algorithm. Section 4 introduces the setups of the test bench and test vehicle. Section 5 presents the results of vehicle tests.

\section{Feature Generation fOr BRUSHLESS ERFS Diagnosis ANd Prognosis}

\subsection{Analytical Model of Brushless DC Motor}

In the brushless ERFS, the low-pressure fuel pump is based on a brushless DC motor. An inverter is used to take a DC electrical input, convert it into a 3-phase electrical output, and send it to the motor. The inverter is in the FTZM. Figure 2 shows the structure of the brushless DC motor and the inverter.

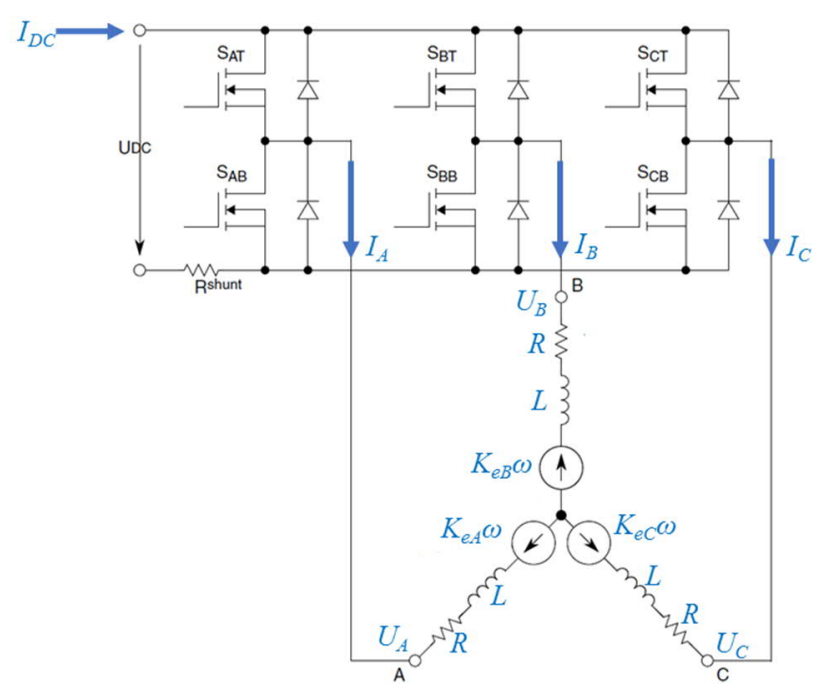

Figure 2. Brushless DC motor and inverter.

At the top of Figure 2, the inverter is represented by the circuit array of six transistors and diodes. DC voltage $U_{D C}$ and current $I_{D C}$ are input to the inverter. A shunt resistor, $\mathrm{R}_{\text {shunt }}$, is used to sense $I_{D C}$. The six transistors $\left(\mathrm{S}_{\mathrm{AT}}, \mathrm{S}_{\mathrm{AB}}, \mathrm{S}_{\mathrm{BT}}\right.$, $\mathrm{S}_{\mathrm{BB}}, \mathrm{S}_{\mathrm{CT}}$ and $\mathrm{S}_{\mathrm{CB}}$ ) are controlled by gate signals in the FTZM.

At the bottom of Figure 2, the circuit of three phases represents the brushless DC motor. The phase voltages are denoted as $U_{A}, U_{B}$ and $U_{C}$, and phase currents as $I_{A}, I_{B}$ and $I_{C}$. Each phase is modeled as a series of a phase resistance $R$, phase inductance $L$, and phase back EMF $\left(K_{e A} \omega, K_{e B} \omega\right.$ and $K_{e C} \omega$, respectively). $\omega$ is the motor speed. $K_{e A}, K_{e B}$ and $K_{e C}$ are the back EMF coefficients of the associated phases, which are trapezoidal.

When the gates in the inverter take turns to be on or off, the phases are activated periodically to maintain the motor rotation. Refer to the work of Prokop and Chalupa (2005) for more details on brushless DC motor and its control.

We ignore the voltage drop at the transistors of the inverter. Based on the work of Moseler and Isermann (2000), the analytical model of a brushless DC motor under a steady state is derived as:

$$
U_{D C} \eta=2 R \operatorname{mean}\left(I_{D C}\right) / \eta+2 K_{e} \operatorname{mean}(\omega)
$$

where $\eta$ is the PWM duty cycle, and $K_{e}$ is the absolute value of the back EMF coefficient when a phase is activated, which is a constant for a trapezoidal back EMF coefficient.

\subsection{Average Phase Current}

In the FTZM of this study, the average phase current (Moseler \& Isermann, 2000) of the activated phase is measured with the shunt resistor. The average phase current is denoted as $I_{A V}$. It satisfies: 


$$
I_{A V}=\operatorname{mean}\left(I_{D C}\right) / \eta
$$

We collect samples of $I_{A V}$ and $I_{D C}$ on a test bench with a pump to validate Equation (2). On the test bench, we measure $\left(I_{A V}\right.$, $\left.I_{D C}, \eta\right)$ under different fuel pressure, pump speed and motor resistance. The measurements are shown in Figure 3. It shows that mean $\left(I_{A V}\right)$ and mean $\left(I_{D C}\right) / \eta$ have a linear correlation, although they are not exactly identical due to the measure error. This error is ignored and has no impact to the diagnostic performance.

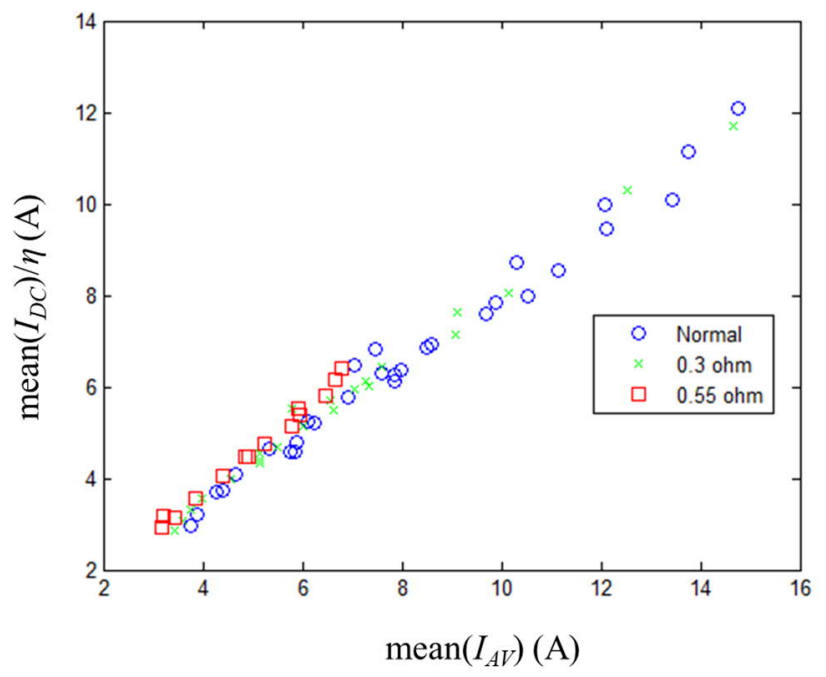

Figure 3. The correlation between $\operatorname{mean}\left(I_{A V}\right)$ and mean $\left(I_{D C}\right) / \eta$. "Normal" means that no increase on the motor resistance is introduced; " $0.3 \mathrm{ohm"} \mathrm{means} \mathrm{that} 0.3$ ohm is added to each phase of the motor; " $0.55 \mathrm{ohm}$ " means that $0.55 \mathrm{ohm}$ is added to each phase of the motor.

With Eq. (2), Eq. (1) becomes:

$$
U_{D C} \eta=2 R \text { mean }\left(I_{A V}\right)+2 K_{e} \text { mean }(\omega)
$$

This is the analytical model with the average phase current.

\subsection{Resistance Estimation}

With the analytical model formulated in Eq. (3), we estimate the equivalent DC resistance of the brushless DC motor:

$$
R=\left[U_{D C} \eta-2 K_{e} \operatorname{mean}(\omega)\right] /\left[2 \operatorname{mean}\left(I_{A V}\right)\right]
$$

Figure 4 shows the results of resistance estimation under three different conditions: normal, $0.3 \mathrm{ohm}$ added to each phase, and $0.55 \mathrm{ohm}$ added to each phase. In reality, a resistance increase of $0.3 \mathrm{ohm}$ is a marginal fault with which the engine may stall after start. A resistance increase of 0.55 $\mathrm{ohm}$ is a more severe fault with which the engine may fail to start. Under each condition, we measure the parameters of $U_{D C}, \eta, \omega$ and $I_{A V}$ of a sample fuel pump on the test bench with different configurations of pump speed and fuel pressure. With these parameters, $R$ is calculated with Eq. (4). In Figure 4, each curve represents the correlation between $R$ and $\omega$ under a given fuel pressure. Figure 4 a) shows that the estimated resistance is in the range between $0.3 \mathrm{ohm}$ and 0.6 ohm under normal condition; Figure $4 \mathrm{~b}$ ) shows that the estimated resistance is in the range between $0.6 \mathrm{ohm}$ and 0.8 $\mathrm{ohm}$ when there is $0.3 \mathrm{ohm}$ added to each phase; Figure $4 \mathrm{c}$ ) shows that the estimated resistance is in the range between $0.9 \mathrm{ohm}$ and $1.2 \mathrm{ohm}$ when there is $0.55 \mathrm{ohm}$ added to each phase. With the value of the estimated resistance, we can determine the resistance increases on the three phases. Please notice that with $0.3 \mathrm{ohm}$ increase the fuel pressure cannot reach $600 \mathrm{kPa}$, and with $0.55 \mathrm{ohm}$ increase it cannot reach $400 \mathrm{kPa}$.

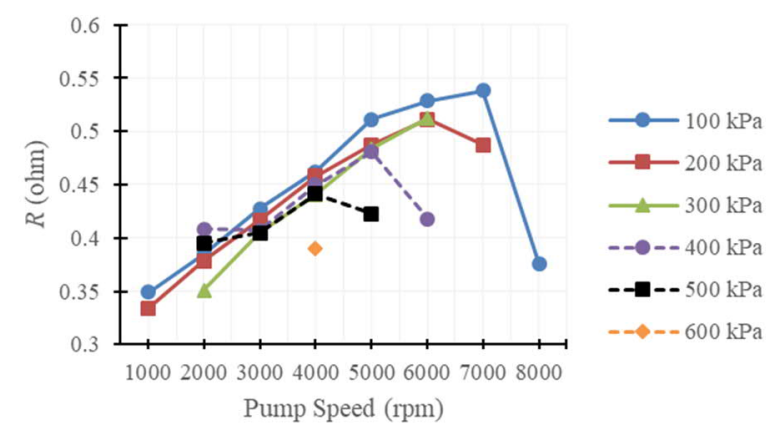

a)

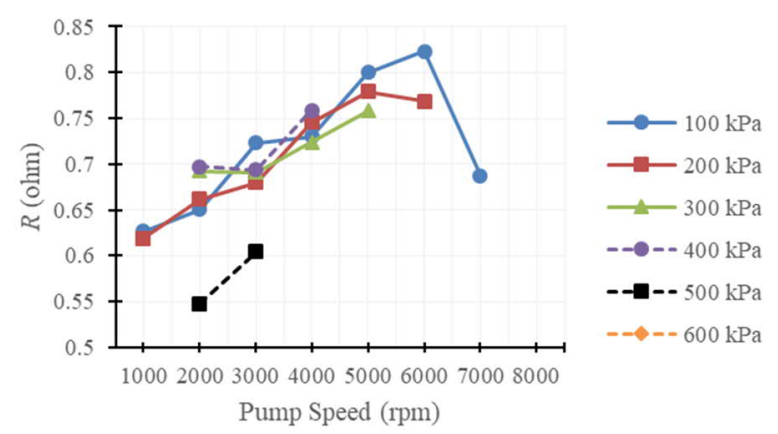

b)

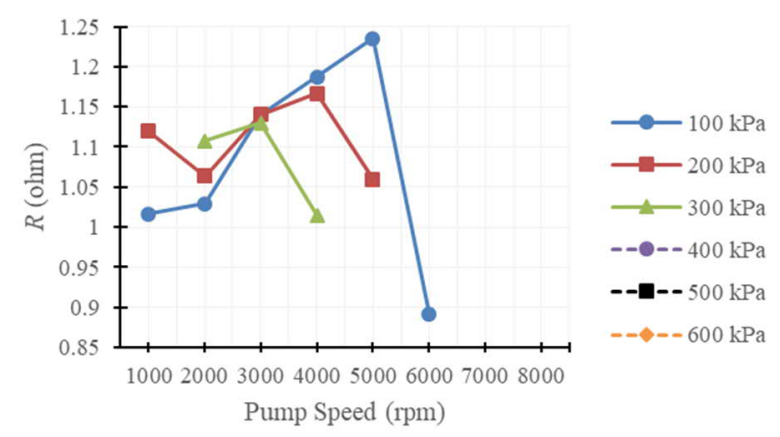

c)

Figure 4. Estimated resistance. a) under normal condition. b) when $0.3 \mathrm{ohm}$ is added to each phase. c) when $0.55 \mathrm{ohm}$ is added to each phase.

\subsection{Estimation of Motor Current Under Normal Condition}

The motor current is correlated to the fuel pressure, since it is proportional to the motor torque. Such a correlation is useful 
when detecting faults such as sensor bias. Figure 5 shows the correlation between the motor current and the fuel pressure of a sample fuel pump under different pump speed.

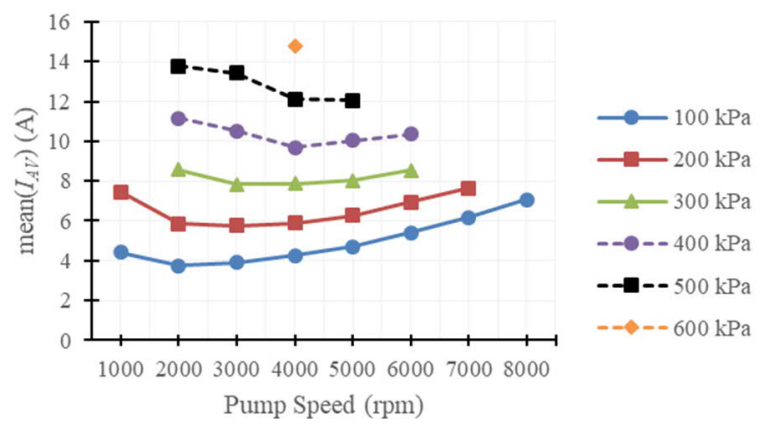

Figure 5. mean $\left(I_{A V}\right)$ under different fuel pressure and pump speed in normal condition.

Each curve in Figure 5 is associated with a given fuel pressure. Comparing to the change of mean $\left(I_{A V}\right)$ over the fuel pressure, the change of mean $\left(I_{A V}\right)$ over the pump speed is relatively small. Samples in Figure 5 are measured under the normal condition. We also measure the current under the conditions of resistance increased on each phase. The results are similar to Figure 5. This means the current is not sensitive to resistance change.

We create a lookup table with the samples in Figure 5. For any given fuel pressure and pump speed, we interpolate the lookup table and determine an estimated current $I_{S S}$ which represents the steady state mean $\left(I_{A V}\right)$ under the normal condition. In Subsection 2.7, we will compare $I_{s s}$ with the actual mean $\left(I_{A V}\right)$. The difference between them indicates that the ERFS is not in a normal condition.

\subsection{Estimation of PWM Duty Cycle Under Normal Condition}

Similar to the estimation of motor current in Subsection 2.4, we also estimate the PWM duty cycle under the normal condition. The characterization of a normal fuel pump collects samples of PWM duty cycle under different configurations of the fuel pressure and pump speed. The characterization results of a sample fuel pump are shown in Figure 6.

Figure 6 shows the correlation of the PWM duty cycle with the fuel pressure and pump speed in the normal condition. The mean of PWM duty cycle increases when the pump speed or fuel pressure increases. In other bench tests with resistance increased on each phase of the motor, it is shown that the PWM duty cycle is higher than that in the normal condition under the same pump speed and fuel pressure.

With the samples shown in Figure 6, we create a lookup table of PWM duty cycle. For any given pump speed and fuel pressure, we interpolate the lookup table to determine the estimated PWM duty cycle $\eta_{s s}$ in the normal condition. In
Subsection 2.7, we will compare $\eta_{s s}$ with the actual $\eta$ to determine if the ERFS is working in a normal condition.

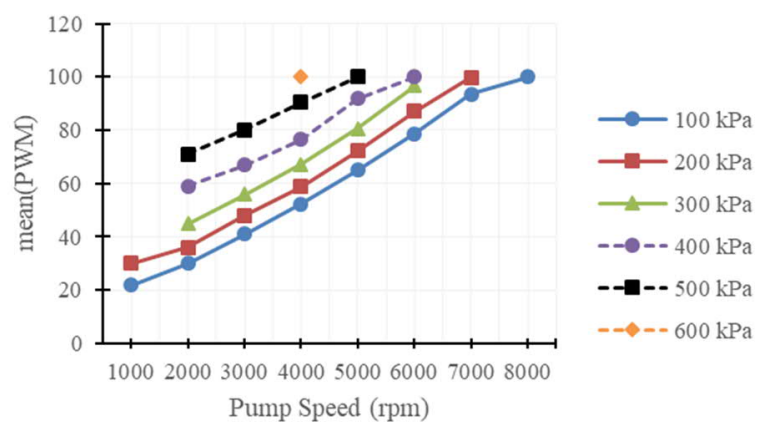

Figure 6. mean(PWM) under different fuel pressure and pump speed in normal condition.

\subsection{Estimation of Pump Speed Under Normal Condition}

For the brushless ERFS, the pump speed can indicate whether the fuel pump is working in a normal condition. Since the analytical model of the pump motor is formulated as a DC model in Eq. (3), we use the same observer in the work of Ghoneim (2013) to estimate the pump speed under the normal condition based on motor voltage and current. The estimated pump speed $\omega_{M}$ will be compared with the actual speed $\omega$ in Subsection 2.7 to determine the working condition of the ERFS.

\subsection{Summary of Feature Signals}

Feature signals are the outputs of the feature generation algorithm of the brushless ERFS DnP. Table 1 lists the features developed in this study for the brushless ERFS DnP. $R, I_{s s}, \eta_{s s}$ and $\omega_{M}$ have been discussed in Subsection 2.3, 2.4, 2.5 and 2.6, respectively.

Table 1. Features generated for the brushless ERFS DnP.

\begin{tabular}{|l|l|}
\hline Feature & Description \\
\hline$R$ & Estimated resistance. \\
\hline$I_{s S}$ & $\begin{array}{l}\text { Estimated average phase current of the pump } \\
\text { in steady state. }\end{array}$ \\
\hline$\eta_{s s}$ & Estimated PWM duty cycle in steady state. \\
\hline$\omega_{M}$ & Estimated pump speed using observer. \\
\hline$\beta_{I}$ & Current error ratio \\
\hline$\beta_{\eta}$ & PWM duty cycle error ratio \\
\hline$\beta_{\omega}$ & Pump speed error ratio \\
\hline$\varepsilon_{\omega}$ & Pump speed error \\
\hline$T_{z \omega}$ & Zero pump speed ratio \\
\hline
\end{tabular}

We calculate $\beta_{I}$ to compare $I_{s s}$ and mean $\left(I_{A V}\right)$. It is the ratio between these two:

$$
\beta_{I}=I_{s s} / \text { mean }\left(I_{A V}\right)
$$

Similarly, we calculate $\beta_{\eta}$ to compare $\eta_{s s}$ and $\eta$ :

$$
\beta_{\eta}=\eta_{s s} / \eta
$$


To compare the pump speed $\omega$ and $\omega_{M}$, we calculate not only their ratio but also the exact error, since the error is a better indicator of faults than the ratio under some faults such as unbalanced resistance increase. This is demonstrated by results presented in Section 5. The ratio is calculated with:

$$
\beta_{\omega}=\omega / \omega_{M}
$$

The error between $\omega$ and $\omega_{M}$ is calculated with:

$$
\varepsilon_{\omega}=\omega-\omega_{M}
$$

Under the fault of unbalanced resistance increase on three phases, the phase resistance in each commutation step is inconsistent. Consequently, the pump speed in each commutation step is inconsistent. The FTZM is not able to synchronize with commutation, which makes the sensing of the pump speed malfunctioning. Speed of 0 value is reported by the FTZM. We count the number of the samples with 0 speed to detect the unbalanced resistance fault.

For every $C_{\omega}$ samples of speed, we count the number of samples with 0 speed. The count is denoted as $C_{z \omega} . T_{z \omega}$ is the ratio of $C_{z \omega}$ over $C_{\omega}$.

$$
T_{z \omega}=100 C_{z \omega} / C_{\omega}
$$

which represents the percentage of the zero speed samples among all the samples.

\subsection{Behaviors of Feature Signals under Faults}

With the feature signals, we test their behaviors under different conditions of the brushless ERFS, including normal and fault conditions.

The faults in this study are balanced resistance increase, unbalanced resistance increase, positive sensor bias and negative sensor bias. The balanced resistance increase represents the resistance with the same amount increased on three phases of the brushless motor. The unbalanced resistance increase represents the increase of resistance are not identical among the three phases. These two faults come from the cases including loose connection, corrosion, wiring or coil issues. The positive and negative sensor bias represent the readings of the fuel pressure are higher and lower than the actual pressure value, respectively. These two faults are from the cases that the fuel pressure sensor is inaccurate.

We observe the behaviors of feature signals with vehicle tests. Details of the test results are presented in Section 5. Table 2 summarizes the feature signal behaviors under different faults compared to under the normal condition. The upwards arrow presents that the feature signal is higher than normal; the downwards arrow presents that the feature signal is lower than normal; the blank cell presents that the feature signal doesn't shown any constant pattern or any difference between faulty and normal behavior.

For the balanced and unbalanced resistance increase, the estimated resistance $R$ is higher than normal. This signature doesn't show under a sensor bias fault. For the bias sensor faults, the current error ratio $\beta_{I}$ is a good indicator. It doesn't show a signature under a resistance increase fault. Also, the current error ratio behaviors oppositely under the negative and positive bias faults.

Table 2. Behaviors of feature signals under different faults.

\begin{tabular}{|l|l|l|l|l|l|l|}
\hline & $R$ & $\beta_{I}$ & $\beta_{\eta}$ & $\varepsilon_{\omega}$ & $\beta_{\omega}$ & $T_{z \omega}$ \\
\hline $\begin{array}{l}\text { Balanced Resistance } \\
\text { Increase }\end{array}$ & $\uparrow$ & & $\downarrow$ & $\downarrow$ & $\downarrow$ & \\
\hline $\begin{array}{l}\text { Unbalanced Resistance } \\
\text { Increase }\end{array}$ & $\uparrow$ & & $\uparrow$ & $\downarrow$ & $\downarrow$ & $\uparrow$ \\
\hline Positive Sensor Bias & & $\uparrow$ & $\uparrow$ & & & \\
\hline Negative Sensor Bias & & $\downarrow$ & $\downarrow$ & & & \\
\hline
\end{tabular}

Since the behaviors are different under different faults, we determine the fault signatures based on these feature signals. In Section 3, we develop the diagnostic and isolation algorithm based on the feature signals.

\section{DiAgNostiC AND ISOlation Algorithm}

With the behaviors of feature signals, a fault diagnostic and isolation algorithm is developed by checking each of the feature signals. Predetermined thresholds are used to determine the ranges of signals, respectively. Figure 7 shows the flow chart of the fault diagnostic and isolation algorithm.

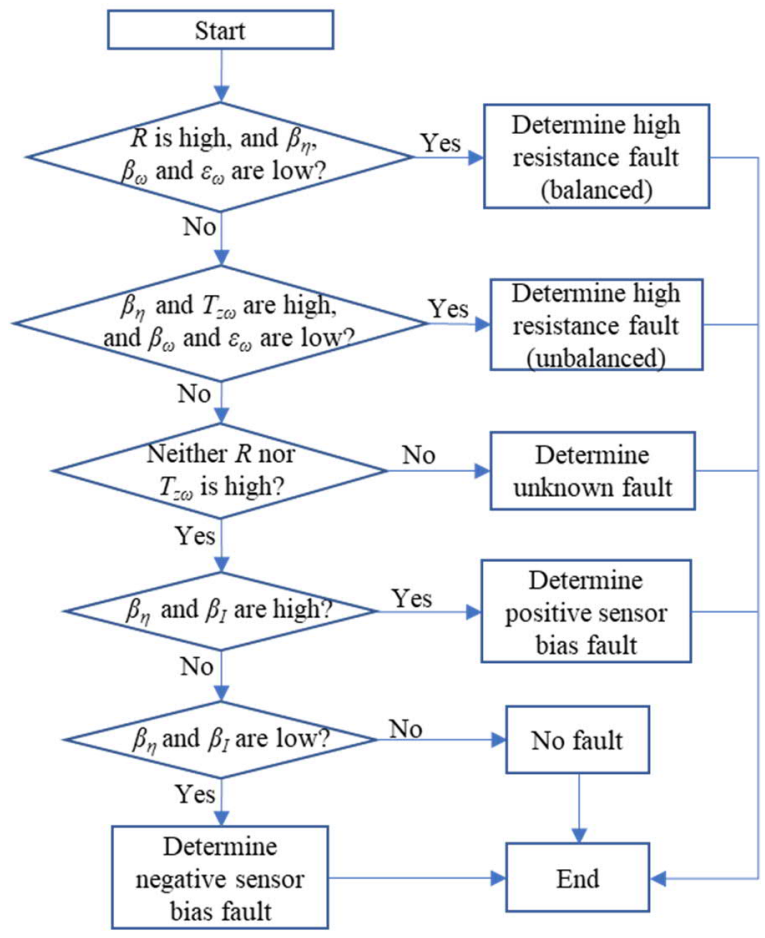

Figure 7. The flowchart of the diagnostic and isolation algorithm.

The algorithm starts with checking the fault of balanced resistance increase. If $R$ is high, and $\beta_{\eta}, \beta_{\omega}$ and $\varepsilon_{\omega}$ are low, 
then a balanced resistance increase is determined. Next, an unbalanced resistance increase is determined if $\beta_{\eta}$ and $T_{z \omega}$ are high, and $\beta_{\omega}$ and $\varepsilon_{\omega}$ are low. Please notice that the condition of an unbalanced resistance increase doesn't exclude the condition that $R$ is high. When the condition of a balanced resistance increase is not satisfied, it means that high $R$, low $\beta_{\eta}$, low $\beta_{\omega}$ and low $\varepsilon_{\omega}$ are not satisfied at the same time. Thus, $R$ may still be high when checking unbalanced resistance increase, and it is implicitly true when $\beta_{\eta}$ and $T_{z \omega}$ are high, and $\beta_{\omega}$ and $\varepsilon_{\omega}$ are low, based on Table 2 . Therefore, we don't need to explicitly check $R$ to determine an unbalanced resistance increase. To determine the sensor bias faults, we first check $R$ and $T_{z \omega}$. If either $R$ or $T_{z \omega}$ is high, we determine an unknow fault, since it doesn't match the behavior of any fault. Under the condition that neither $R$ nor $T_{z \omega}$ is high, if $\beta_{\eta}$ and $\beta_{I}$ are high, a positive sensor bias is determined; if $\beta_{\eta}$ and $\beta_{I}$ are low, a negative sensor bias is determined; if $\beta_{\eta}$ and $\beta_{I}$ are in the normal range, a normal condition is determined. With this algorithm, the diagnostic results are generated for the brushless ERFS.

\section{Test Bench Setup and Test Vehicle Setup}

A test bench is built to control a brushless fuel pump to operate under different conditions, which is used to measure and collect the data of signals discussed in Section 2.

Figure 8 shows the setup of the brushless fuel pump test bench. A brushless fuel pump module is mounted in a fuel tank. A fuel feed pipe is connected to the fuel nozzle on the fuel pump module, and sends the stoddard back to the tank. A fuel valve on the feed pipe is used to control the fuel pressure and flow rate coming out of the pump. A fuel pressure sensor and a fuel flow sensor are installed on the pipe to measure the fuel pressure and flow rate $F$. Their measurements are read by Micro AutoBox 1, and the data are collected by Laptop 1.

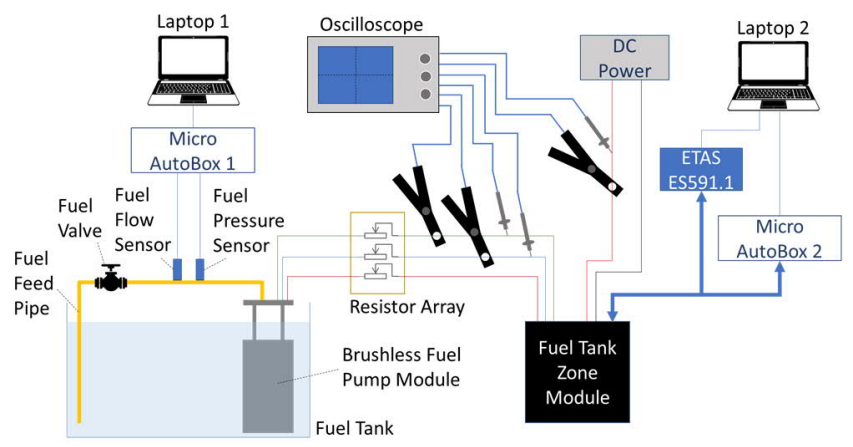

Figure 8. Brushless fuel pump test bench.

The brushless fuel pump module is driven by the fuel tank zone module through the 3-phase power wires. A resistor array is installed on the power wires to adjust the resistance on the 3 phases independently. The fuel tank zone module is powered by a DC power supply.
An oscilloscope is used to measure and collect the data of the DC current $I_{D C}$, DC voltage $U_{D C}$, two phase currents, and two phase voltages.

Laptop 2 sends the commanded pump speed, which goes to the FTZM through Micro AutoBox 2. In addition, the actual pump speed $\omega$ and the average phase current $I_{A V}$ sensed by the FTZM are read by Micro AutoBox 2, and their data are collected by Laptop 2 .

An ETAS ES591.1 is connected to the FTZM through the CAN bus to read the PWM duty cycle $\eta$ measured by the FTZM. Its data is collected by Laptop 2.

In addition, a test vehicle is built with the feature generation algorithm implemented on-board in real-time. With the test vehicle, we fine-tune and validate the algorithm with the actual measurements in the vehicle.

Figure 9 shows the test vehicle setup. An instrumented ECM is installed to propagate internal signals, including the DC voltage $U_{D C}$, average phase current $I_{A V}$, sensed pump speed $\omega$, desired pump speed, sensed fuel pressure, desired fuel pressure, etc. An ETAS ES592.1 is used to receive the internal signals from the ECM through an ETK cable, and enables a laptop to collect them.

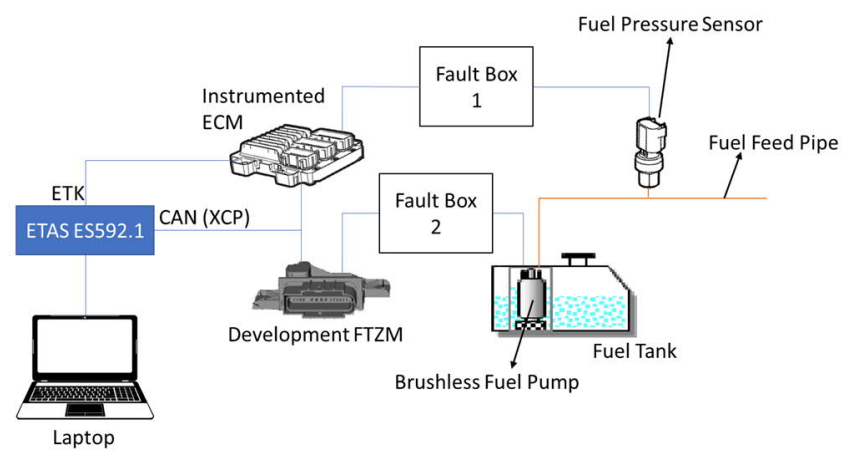

Figure 9. Test vehicle setup for brushless fuel DnP.

The ETAS ES592.1 reads the PWM duty cycle $\eta$ from a development FTZM. The communication between the ECM and FTZM is monitored by the ETAS ES592.1 through a CAN cable. The laptop collects data of the PWM duty cycle through the ETAS ES592.1.

Fault Box 1 is connected between the ECM and fuel pressure sensor. It adjusts the voltage of the sensor to introduce a sensor bias. Fault Box 2 is connected between the FTZM and brushless fuel pump. It adjusts the resistance of the three phases independently.

\section{RESUltS}

In previous sections, we discuss the details of the features for the brushless ERFS DnP and the diagnostic algorithm. In this section, we discuss the results of these algorithms. We collect data from the test vehicle with fault injection. The data are processed with the algorithms. 
We inject four types of faults discussed in Subsection 2.8. For resistance increase, we add additional resistance to the phases accordingly. For the sensor bias, we introduce voltage offsets on the fuel pressure sensor, respectively.

The test conditions are in 11 categories: normal, balanced resistance increase of $0.214 \mathrm{ohm}$, balanced resistance increase of $0.375 \mathrm{ohm}$, unbalanced increase of $0.214 \mathrm{ohm}$ on two phases, unbalanced increase of $0.375 \mathrm{ohm}$ on two phases, unbalanced increase of $0.214 \mathrm{ohm}$ on one phase, unbalanced increase of $0.375 \mathrm{ohm}$ on one phase, positive bias of $0.5 \mathrm{~V}$ on the fuel pressure sensor, positive bias of $1 \mathrm{~V}$ on the fuel pressure sensor, negative bias of $0.5 \mathrm{~V}$ on the fuel pressure sensor, and negative bias of $1 \mathrm{~V}$ on the fuel pressure sensor. For each condition, we test under three driving conditions: idling, driving on a flat road, and driving on hills. Therefore, we test the algorithms in 33 conditions in total.

\subsection{Balanced Resistance Increase}

This subsection presents the results under the fault of balanced resistance increase of $0.214 \mathrm{ohm}$ under the flat road driving condition. The results are showing in Figure 10. The blue solid curve in each sub figure presents the behavior of the corresponding feature signal over time under the normal condition; the green dash curve presents the behavior under the balanced resistance increase. We add $0.214 \mathrm{ohm}$ on each of the three phases to introduce a fault of the balanced resistance increase.
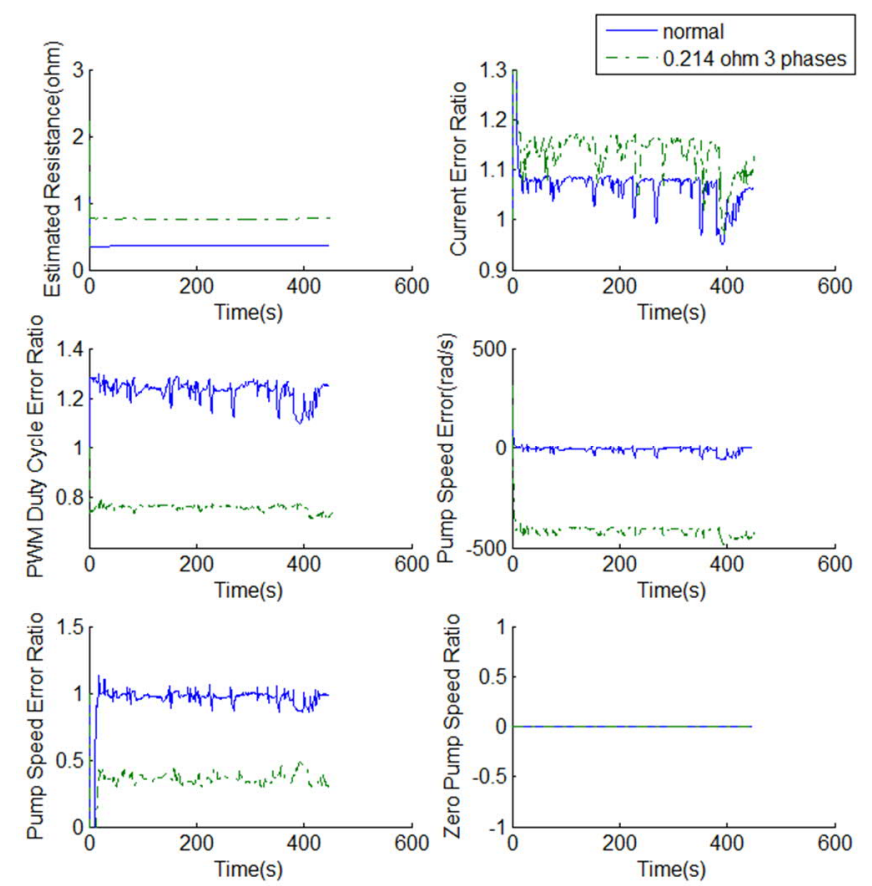

Figure 10. Feature behaviors under balanced resistance increase.

The experimental results show that some feature signals change under the fault condition compared to under the normal condition. The estimated resistance $R$ is increased under the introduced fault. The current error ratio $\beta_{I}$ is higher than normal, because the actual phase current is smaller than the normal value due to the resistance increase. The PWM duty cycle error ratio $\beta_{\eta}$ decreases under the fault, because the fuel pump works harder, and the actual PWM duty cycle is higher than in the normal condition. The pump speed increases under the fault. Therefore, the pump speed error $\varepsilon_{\omega}$ is negative, and the pump speed error ratio $\beta_{\omega}$ is smaller than 1. The zero pump speed ratio $T_{z \omega}$ is zero under both the normal and balanced resistance increase conditions, because the pump speed is constant during commutations.

Figure 10 shows $\beta_{I}$ and $\beta_{\eta}$ are not 1 in the normal condition. Based on Eq. (5) and (6), the results represent that the current and PWM duty cycle are higher in the bench than in the vehicle under the normal condition. This is due to the difference between the hydraulic systems in the bench and vehicle. Compared to the vehicle, the bench has a bigger pressure loss from the pump to the pressure sensor. This difference doesn't impact the capability of DnP, since we use the change of $\beta_{I}$ and $\beta_{\eta}$ under faulty and normal conditions, instead of their exact values.

\subsection{Unbalanced Resistance Increase}

This subsection discusses the results under the fault of unbalanced resistance increase of $0.375 \mathrm{ohm}$ under the flat road driving condition. Figure 11 shows the results.
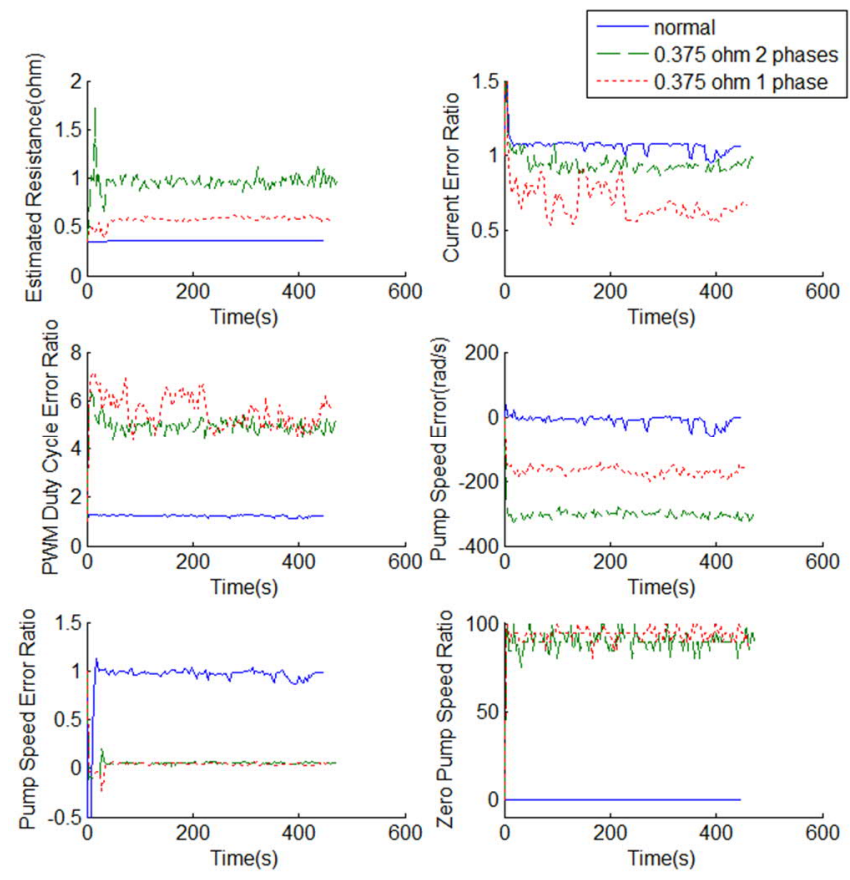

Figure 11. Feature behaviors under unbalanced resistance increase.

In Figure 11, the blue solid curve in each sub figure presents the behavior under the normal condition; the green dash curve 
presents the behavior when two phases have resistance increased, each with a $0.375 \mathrm{ohm}$ increase; the red dotted curve presents the behavior when one phase has a $0.375 \mathrm{ohm}$ resistance increase.

Under the unbalanced resistance increase, the estimated resistance $R$ is higher than normal. Since the pump speed is inconsistent in one commutation cycle, the FTZM loses control on the pump speed. The pump speed is lower than normal. Thus, the pump speed error $\varepsilon_{\omega}$ and error ratio $\beta_{\omega}$ are lower than normal. Low speed also causes the phase current higher than normal and the PWM duty cycle lower than normal. This behavior of the phase current is different from that under the balanced resistance increase. Consequently, the current error ratio $\beta_{I}$ is lower than normal, and the PWM duty cycle error ratio $\beta_{\eta}$ is higher than normal. Due to the zero speed samples, the zero pump speed ratio $T_{z \omega}$ is greater than 0 .

\subsection{Fuel Pressure Sensor Bias}

This subsection discusses the results under the faults of the fuel pressure sensor bias of $1 \mathrm{~V}$ under the flat road driving condition. Figure 12 shows the results. The blue solid curve presents the behavior under the normal condition; the green dash curve presents the behavior under the positive bias of $1 \mathrm{~V}$ on the pressure sensor; the red dotted curve presents the behavior under the negative bias of $1 \mathrm{~V}$ on the pressure sensor.
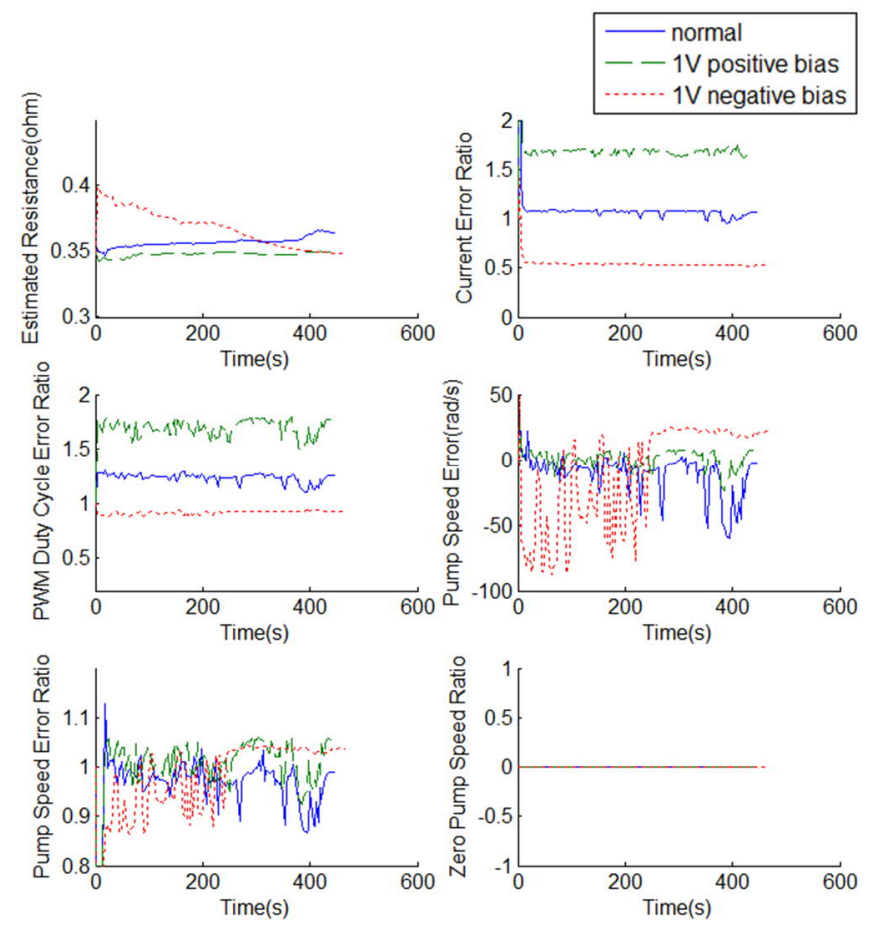

Figure 12. Feature behaviors under the fuel pressure sensor bias faults.

In Figure 12, the estimated resistance $R$ under the bias faults is in the range between $0.35 \mathrm{ohm}$ and $0.4 \mathrm{ohm}$, which is considered as a normal range. The estimated current is associated with the pressure. With a positive bias, the sensed pressure is higher than the actual pressure. Thus, the estimated current is higher than the actual value. The current error ratio $\beta_{I}$ is higher than normal. Similarly, the current error ratio $\beta_{I}$ is lower than normal under the negative bias fault.

Under the positive bias fault, the actual PWM duty cycle is lower than normal, since the system thinks the pressure reaches the demand, and stops increase the PWM duty cycle. The estimated PWM duty cycle is higher than normal based on the lookup table. Therefore, the PWM duty cycle error ratio $\beta_{\eta}$ is higher than normal. Similarly, the current error ratio $\beta_{\eta}$ is lower than normal under the negative bias fault.

The absolute value of the speed error $\varepsilon_{\omega}$ under the senor faults is much smaller than the absolute value of the speed error under resistance increase faults. The speed error ratio $\beta_{\omega}$ is close to 1. $\varepsilon_{\omega}$ and $\beta_{\omega}$ are much more unstable under the negative bias fault than the positive bias. This is because the pump is more unstable under higher duty cycle. The zero pump speed ratio $T_{z \omega}$ is 0 , since there isn't any zero speed sample.

\subsection{Diagnostic Results}

In addition to the results discussed above, we summarize the final diagnostic results in all the 33 test conditions in this subsection. The three tests under normal condition all generate "no fault" diagnostic results.

Tests with balanced resistance increase of $0.375 \mathrm{ohm}$ generate the "high resistance fault, balanced" results under flat road driving and hill driving. However, the "high resistance fault, unbalanced" results are generated under idling. This corner case is due to that 1) $T_{z \omega}$ is not always 0 (since $0.375 \mathrm{ohm}$ is relatively high so that the FTZM has difficulty to drive the motor at a steady speed), and 2) PWM duty cycle is lower than normal (since the pump speed is low during idling). In this case, the motor behaviors similarly to the condition of unbalanced resistance increase. This corner case needs additional algorithm to handle in the future.

For all the three tests with balanced resistance increase of $0.214 \mathrm{ohm}$, the speed is consistent, and the "high resistance fault, balanced" results are generated.

The test is incomplete under the unbalanced resistance increase, $0.375 \mathrm{ohm}$ on one phase, hill driving, because the fault is big and stalls the engine. For all other 11 tests under unbalanced resistance increase condition, the "high resistance fault, unbalanced" diagnostic results are generated.

For all the 12 tests of positive and negative sensor bias faults, the diagnostic results "positive sensor bias" and "negative sensor bias" are generated, respectively. 


\section{Conclusion}

Based on the analytical model of the brushless DC motor, we develop a set of feature signals which can represent the behaviors of the brushless ERFS under different conditions. We develop the fault diagnostic and isolation to check feature signals and determine the exact fault condition. The algorithms are tested in a series of vehicle tests, and show good capability of identifying different faults. The DnP algorithms can protect customer from severe vehicle failures. They also provide diagnostic data during the service process to better diagnose the root causes of issues, which can reduce incorrect repairs. Moreover, they support the engineering to address production issues by providing the data and prognosis/diagnosis results of vehicles in the field.

In the future, more validation will be performed on a bigger fleet of test vehicles. Additional algorithm will be developed to handle corner cases, such as the balanced resistance increase of a high amount.

\section{NOMENCLATURE}

$\begin{array}{ll}\beta_{I} & \text { current error ratio } \\ \beta_{\eta} & \text { PWM duty cycle error ratio } \\ \beta_{\omega} & \text { pump speed error ratio } \\ \varepsilon_{\omega} & \text { pump speed error } \\ \eta & \text { PWM duty cycle } \\ \eta_{s s} & \text { estimated PWM duty cycle under normal } \\ I_{A}, I_{B}, I_{C} & \text { condition } \\ I_{A V} & \text { phase current of the brushless motor } \\ I_{D C} & \text { average phase current } \\ I_{s S} & \text { DC current at the inverter input } \\ K_{e} & \text { estimated steady state phase current under } \\ & \text { normal condition } \\ K_{e A}, K_{e B}, K_{e C} & \text { absolute value of back EMF coefficient } \\ R & \text { when a phase is activated } \\ T_{z \omega} & \text { back EMF coefficients of each phase } \\ U_{A}, U_{B}, U_{C} & \text { (estimated) phase resistance } \\ U_{D C} & \text { zero pump speed ratio } \\ \omega & \text { phase voltage of the brushless motor } \\ \omega_{M} & \text { DC voltage at the inverter input } \\ & \text { motor speed }\end{array}$

\section{REFERENCES}

Awadallah, M. A., Morcos, M. M., Gopalakrishnan, S., \& Nehl, T. W. (2005). A neuro-fuzzy approach to automatic diagnosis and location of stator inter-turn faults in CSI-Fed PM brushless DC motors. IEEE Transactions on Energy Conversion, vol. 20 (2), pp. 253259. doi:10.1109/TEC.2005.847976

Ghoneim, Y. A. (2013). State of health indicator for a vehicle fuel delivery system. US Patent 8,473,147.

Ghoneim, Y. A. (2014). Fault isolation in electronic returnless fuel system. US Patent 8,770,015.
Ghoneim, Y. A., \& Howell, M. N. (2016). Method and apparatus for on-board/off-board fault detection. US Patent Application 14/423,816.

Lu, C., Wang, Y., Ragulskis, M., \& Cheng, Y. (2016). Fault diagnosis for rotating machinery: A method based on image processing. PLoS One, vol. 11 (10). doi:10.1371/journal.pone.0164111

Moseler, O., \& Isermann, R. (2000). Application of modelbased fault detection to a brushless DC Motor. IEEE Transactions on Industrial Electronics, vol. 47 (5), pp. 1015-1020. doi: 10.1109/41.873209

Prokop, L., \& Chalupa, L. (2005). 3-phase BLDC motor control with sensorless back EMF zero crossing detection using 56F80x. Freescale Semiconductor, Chandler, AZ, USA, Appl. Note AN1913, Rev, 3.

Sun, J., Chai, Y., Su, C., Zhu, Z., \& Luo, X. (2014). BLDC motor speed control system fault diagnosis based on LRGF neural network and adaptive lifting scheme. Applied Soft Computing, vol. 14, pp. 609-622. doi:10.1016/j.asoc.2013.10.010

Tashakori, A., \& Ektesabi, M. (2013). Fault diagnosis of inwheel BLDC motor drive for electric vehicle application. 2013 IEEE Intelligent Vehicles Symposium (IV), June 23-26, Gold Coast, QLD, Australia. doi:10.1109/IVS.2013.6629585

Tax, D. M. J., Ypma, A., \& Duin, R. P. W. (1999). Pump failure detection using support vector data descriptions. In Hand, D. J., Kok, J. N., \& Berthold, M. R. (Eds.), Advances in intelligent data analysis. IDA 1999. Lecture notes in computer science, vol. 1642 (pp. 415-425). Berlin, Heidelberg: Springer.

Wen, L., Li, X., Gao, L., \& Zhang, Y. (2018). A new convolutional neural network-based data-driven fault diagnosis method. IEEE Transactions on Industrial Electronics, vol. 65 (7), pp. 5990-5998. doi: 10.1109/TIE.2017.2774777

\section{BIOGRAPHIES}

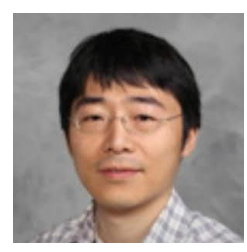

Yao Hu received a B.Sc. in electrical information science and technology from Fudan University, Shanghai, China, in 2004. He received a M.Eng. in system and circuit from Shanghai Institute of Technical Physics, Chinese Academy of Sciences, Shanghai, China, in 2007. He received a Ph.D. in electrical engineering from University of Kentucky, Lexington, KY, in 2013. He has been working at General Motors Global R\&D Center, Warren, MI, since 2016, and currently holds the senior researcher position in the vehicle system research lab. His research interests include vehicle health management, perception, autonomous driving, deep learning and data analytics. 